\title{
Mendeley: Creating Communities of Scholarly Inquiry Through Research Collaboration
}

\author{
Holt Zaugg \\ Brigham Young University - Provo, holt_zaugg@byu.edu \\ Isaku Tateishi \\ Brigham Young University - Provo \\ Daniel L. Randall \\ BYU \\ Richard E. West \\ $B Y U$
}

Follow this and additional works at: https://scholarsarchive.byu.edu/facpub

Part of the Library and Information Science Commons

\section{Original Publication Citation}

Holt Zaugg, Richard E. West, Isaku Tateishi, Daniel L. Randall, Mendeley: Creating Communities of Scholarly Inquiry Through Research Collaboration, TechTrends, January 2011, Volume 55, Issue 1, pp 32-36, Springer US, DOI: 10.1007/s11528-011-0467-y

\section{BYU ScholarsArchive Citation}

Zaugg, Holt; Tateishi, Isaku; Randall, Daniel L.; and West, Richard E., "Mendeley: Creating Communities of Scholarly Inquiry Through Research Collaboration" (2011). Faculty Publications. 1633.

https://scholarsarchive.byu.edu/facpub/1633

This Peer-Reviewed Article is brought to you for free and open access by BYU ScholarsArchive. It has been accepted for inclusion in Faculty Publications by an authorized administrator of BYU ScholarsArchive. For more information, please contact ellen_amatangelo@byu.edu. 
Mendeley: Creating Communities of Scholarly Inquiry Through Research Collaboration

\author{
Holt Zaugg \\ Richard E. West \\ Isaku Tateishi \\ Daniel L. Randall
}

\begin{abstract}
Mendeley is a free, web-based tool for organizing research citations and annotating their accompanying PDF articles. Adapting Web 2.0 principles for academic scholarship, Mendeley integrates the management of the research articles with features for collaborating with researchers locally and worldwide. In this article the features of Mendeley are discussed and critiqued in comparison to other, similar tools. These features include citation management, online synchronization and collaboration, PDF management and annotation, and integration with word processing software. The article concludes with a discussion of how a social networking tool such as Mendeley might impact the academic scholarship process.
\end{abstract}

Keywords: social networking, research, online community, Web 2.0, citation management. 


\section{Mendeley: Creating Communities of Scholarly Inquiry Through Research Collaboration}

Social networking Web 2.0 technologies have grown increasingly popular and, for many people, have changed the way we listen to music (e.g. Last.fm), seek professional opportunities (e.g. Linkedin), share information about our lives with family and friends (e.g. Facebook and Flickr), or even find and share web-based resources (e.g. Diigo). Web 2.0 technologies can also create new opportunities for improvement in professional areas such as medicine (Eysenbach, 2008). Following this trend, social networks might provide many benefits to academic researchers, including assistance in finding similar research articles around particular topics, identifying colleagues for potential collaboration, disseminating one's own research, and developing a clearer understanding of which studies have had the most impact in a particular field.

Thus far, social networks built around academic research have not become widespread, perhaps for two reasons. First, researchers have little time for another social network unless its functionality benefits them and improves their research. Second, academics and researchers might hesitate to openly post their developing research lest they get pre-empted by another researcher or receive public criticism for their still-evolving research. Thus, a social network centered on research would need to improve the functionality of one's own research and also allow control over how and to whom information is shared.

Mendeley, a free open-source tool available at http://mendeley.com, seeks to address these concerns while building a robust scholarly social network using a model based on Last.fm (Henning \& Reichelt, 2008; Hoyt, Reichelt \& Henning, 2009). As a social network connecting music lovers, Last.fm identifies music, not people, as the key element in a user's social network. 
It focuses on which music is listened to, how often, and by whom. In this manner Last.fm automatically connects music listeners with those who have similar interests, without the users needing to expend time and energy developing their network.

Mendeley uses a similar strategy. Mendeley provides intriguing features for developing one's professional research network. Mendeley consists of two main programs: a desktop citation and PDF manager and an online companion that can also manage citations while sharing user-defined pieces of information about one's research with others (Mendeley, 2009). Similar to the Last.fm model, Mendeley focuses on researchers' libraries instead of on the researchers themselves. Thus, networks can be formed around strands of research and specific articles. To do this, Mendeley aggregates articles chosen by a researcher, counts how often those articles are read, and connects this researcher with compatible colleagues or additional articles based on key words and information about previous article usage. This may help researchers begin conversations and collaboration with others interested in the same research.

The off-line version of Mendeley is an effective and user-friendly citation tool competing with tools such as Endnote, Refworks, and Zotero while incorporating PDF management and annotation features found in tools such as Sente and Papers. This desktop program can be used independently or together with the online version. This allows researchers to keep documents on their computers that can be synched (or never synched) with the online server. Combined with its online companion, Mendeley is a time-saving free tool for researchers, creating value regardless of how much the social networking potential of the tool is exploited.

In this article, we first review Mendeley's features, discussing some of the tool's positive and negative aspects. We then compare its feature set to similar tools and conclude with a discussion about its social networking potential for researchers. 


\section{Review of Mendeley Features}

Mendeley provides several ways for users to store bibliographical information they wish to manage. Once it is installed, researchers manually create a reference entry, or they import major bibliographical data files, such as BibTex, EndNote XML, and RIS, to create multiple entries at once. The application can also import PDF files and automatically extract bibliographical information from the PDF metadata, such as keywords and cited references, to create the bibliographic entry. Imported files are held in a "needs review" folder until the researcher verifies this bibliographic information. However, depending on the quality of the data source, sometimes there is missing metadata which can easily and quickly be corrected in the document details. Mendeley can also monitor any selected folders on a computer's hard drive and automatically import new PDF files placed into these folders. Finally, like other citation managers, users can save bibliographical information from within most academic search engines (e.g., ScienceDirect, Google Scholar, EBSCO, and JSTOR etc.) directly to Mendeley.

Mendeley's Web Importer works with most popular browsers, including Chrome, and a simple click when viewing an article online allows users to select and save the reference details and associated PDF files into the Mendeley library. These citations can be added to multiple collection folders or organized by assigning tags. Additionally, information in other citation managers, (i.e. Zotero, EndNotes, and RefWorks) can be imported into the Mendeley library. Researchers who want to continue using Zotero when online can set up Zotero synching so that new Zotero references show up automatically in the Mendeley database. Users can search and filter their references by author, author's keywords, the user's own custom tags, and the names of the journals (see Figure 1). 
Insert Figure 1 About Here

\section{Online Synchronization and Collaboration}

Mendeley's online functionality provides another major advantage. With $500 \mathrm{MB}$ of free document space, the bibliographical information, notes, tags, and PDF files from the Mendeley Desktop program are synchronized to the researcher's online Mendeley library. While online, researchers can edit information, create or delete a new entry, and download PDF files. The result is access anywhere, anytime to one's citations and documents. All online changes can be automatically or manually synchronized with the user's desktop version of the Mendeley software. In addition, users can share a specific folder or collection with other invited Mendeley users. This function enables cooperative team research (CTR) for sharing bibliographies and PDF files. Team members may add and edit references and upload PDF files to the folder. Any new information in the shared folder is synchronized with the desktop application of all CTR members. Finally, the Mendeley website allows researchers to find others with similar research interests so researchers can connect, share ideas, ask questions, or start a discussion (see Figure 2).

Insert Figure 2 About Here

\section{PDF Management and Annotation}

Besides organizing citations, Mendeley is a strong PDF management and annotation system. Based on the user's preferences, Mendeley creates a hierarchical folder structure on the user's hard drive in which the PDF files are placed. Documents added to the library are copied into this folder structure from the original source without deleting or altering the originals. 
Within the library, researchers organize their files within personal folders (called library collections), and they can assign stars to important files and mark which PDF files have been read. The library also provides a simple text editor where researchers can add tags and notes about the source as a whole. A weakness of Mendeley is that the researcher can only assign one star to an article, rather than using a 5-star rating system which makes it more difficult to keep track of which articles are more critical than others for particular research reports.

The Mendeley Desktop PDF reader allows researchers to view and highlight text and to add "sticky notes" or annotations (see Figures 3 and 4). Multiple PDF documents can be opened in different tabs within a single Mendeley window. An arrow button allows users to select text to copy and paste into either the notepad or any other text document. However, for some articles, this text does not copy correctly, but instead copies as symbols and punctuation marks (e.g. i î̀y, e, syx $\square \square \square \square \square \square$ ). Unlike some PDF annotation tools, Mendeley does not currently offer other annotation options such as the ability to underline text or draw circles or rectangles around passages. However, an easy option allows users to open a file externally in a morepowerful PDF annotation tool (such as Adobe Acrobat) and make the annotations there. Those annotations are then viewable in Mendeley. Annotations created within Mendeley are only viewable within Mendeley, but an export feature allows users to create a new PDF containing the annotations. An added feature enables users to synchronize their annotations with the web-based portion of Mendeley, offering the option for CTR members to annotate files in shared collections. Currently, Mendeley does not have optical character recognition (OCR) capability, so scanned documents must be annotated in other programs.

Insert Figure 3 About Here 
Insert Figure 4 About Here

\section{Integration with Word Processors}

Like most citation management tools, Mendeley includes a Microsoft Word plug-in that can insert bibliographic information into a document. Clicking the "Insert Citation" button in Word allows users to view the Mendeley Library and to select one or more citations to place in their document. However, the Mendeley Desktop application needs to be open for this feature to work properly. Once a citation has been added to the document, if it is altered in the Mendeley library (i.e. to correct a misspelled name), clicking the refresh button in Word updates the references. The plug-in also allows users to easily switch the citation style. Mendeley tracks the citations used and generates a full bibliography. Word processing integration works for both Windows, Mac (Word 2008 only), and OpenOffice.

\section{Comparison with Other Management Applications}

At their core, citation management programs have much in common, with a major difference being whether they primarily function off-line or online (although this distinction is becoming more transparent in the most recent versions). Mendeley excels by being very capable in both modes. For convenience during web searches, the Mendeley web importer works adequately, although it requires a few extra clicks to see the citations, unlike Zotero, which is always accessible in the bottom pane of a Firefox browser. However, Mendeley can be implemented with more browsers than Zotero. Its desktop companion seems powerful enough to handle a large workload, as one of the authors recently utilized it for a major literature review. 
The ability to organize and find citations by multiple criteria seems faster and more responsive than in other programs.

A major advantage of Mendeley is the inclusion of its very capable PDF annotation and management features. Integrating PDF annotation/management with citation management allows researchers to keep all of their notes, citations, and documents in one place. One of the authors previously used Sente for PDF management and Zotero for online web searches/citation management. In his opinion, Mendeley incorporates most of the convenient and powerful PDF management features of a program like Sente, but is easier and more user-friendly.

Finally, Mendeley adds significant value over other programs because it incorporates research statistics. As the user base grows, Mendeley can report how often articles are saved by different users and how articles are being tagged. This enables two important features. First, it creates a useful list of keywords relating to different articles. Second, it enables the researcher to see how often different articles are being read, or at least accessed. This has the potential to improve upon popular citation indices that rate an article's popularity only by how often it is cited. Mendeley's approach potentially gives a truer sense of an article's impact by showing how often an article is accessed or looked at. Of course, these statistics are limited by how large the Mendeley user base is, how effectively these users incorporate Mendeley into their regular research practice, and how closely the Mendeley user base approximates the characteristics of one's own academic peers. Still the concept is useful, and it provides one more piece of evidence about an article's comparative impact in a particular field.

\section{Research Collaboration Through Social Networking}

How a researcher chooses to use Mendeley determines the ultimate benefits. As mentioned before, Mendeley desktop may be used by a single researcher as a data management 
tool. However, the real power of Mendeley lies in the potential to collaborate, either within a known group or team or with unknown researchers.

A researcher may set up a research group with fellow collaborators. In this case the data management extends through the Internet to allow all group members access to articles. Each member may add to this pool or draw from the pool for similar research or to gather information for lectures or conference presentations. The group is closed to outside participation and sharing. In this way research is protected and disseminated as the researcher decides.

Sharing research among all Mendeley users allows Mendeley to match research articles used and accessed by one researcher with another. If a researcher has accessed a particular article several times, Mendeley uses the key words and topics to suggest similar articles and researchers. Each researcher has the option of pursuing this recommended new contact or not.

This has several positive benefits. First, those researching a particular area may find additional resources more efficiently through these contacts. Second, researchers conducting parallel or adjacent research may be identified. Collaboration may substantiate research findings quicker or expand those findings more efficiently and expeditiously. Third, through contact with other researchers, one may identify key questions for further research or research areas that have led to dead ends. These benefits may be able to improve research efficiency.

\section{Conclusion}

Ultimately, choosing a citation management program depends on the researcher's preferences. In considering the options, a researcher could reflect on the following questions: What kind of word processor do I typically use? How much integration do I need between a citation program and a word processor? What kinds of collaborative features do my co-authors and I require? Do I primarily work with my citations off-line or online, or do I need both? How 
do I like to mark up/organize/read my PDFs? Which browser do I prefer? How willing am I to share and collaborate with others at all stages of my research?

For many, Mendeley offers a compelling list of features that are highly usable and functional. It is user friendly for technological immigrants looking to update their research skills, yet is saavy enough for Web 2.0 natives seeking to explore the potential that social networks might have for benefiting their scholarship. It offers the possibility to align and collaborate on research projects throughout the world while allowing researchers to determine their level of openness and collaboration. Incorporating this social networking approach to academic research also allows for peer review and feedback much earlier in the research process. It helps establish researcher expertise in more rapid, dynamic ways before an article is submitted to a potential journal. As Greenhow, Robelia, and Hughes (2009) said, "Validity of knowledge in Web 2.0 environments is established through peer review in an engaged community, and expertise entails understanding disputes and offering syntheses widely accepted by the community" In short, Mendeley takes the genre of research citation management software to a new, and potentially powerful level, and offers a glimpse of how academic scholarship may adapt to the affordances of an evolving Internet.

\section{BRIEF AUTHOR BIOGRAPHIES}

Holt Zaugg (zauggh@ gmail.com) is a doctoral student at Brigham Young University in the Educational Inquiry, Measurement and Evaluation program. His current research focuses on teaching cross-cultural competencies and virtual communication skills to engineering students working on global, virtual teams. 
Richard E. West (http://byuipt.net/wests/rick ) is an assistant professor at Brigham Young University. He has taught online and face-to-face courses on technology integration for preservice teachers, program evaluation, and instructional design. He has published articles related to communities of innovation/learning/practice, technology integration, distance learning, and evaluation.

Isaku Tateishi is a doctoral candidate at Brigham Young University in the Instructional Psychology and Technology program. He currently researches the impact of group collaboration on creative thinking ability and how cross-cultural virtual collaboration improves competencies necessary to effectively work in the global working environment.

Daniel L. Randall is a master's student in Instructional Psychology and Technology at Brigham Young University. His areas of interest include collaborative innovation, instruction through story telling and the use of video in instruction. 


\section{References}

Eysenbach, G. (2008). Medicine 2.0: Social networking, collaboration, participation, apomediation, and openness. Journal of Medical Internet Research. 10(3): e22. doi: 10.2196/jmir.1030.

Greenhow, C.; Robelia, B.; \& Hughes, J. E. (2009). Web 2.0 and classroom research: What path should we take? Educational Researcher, 38(4): 246-259.

Henning, V. \& Reichelt J. (2008) Mendeley - A last.fm for research? 2008 IEEE Fourth International Conference on eScience, 1-2.

Hoyt, J. J., Reichelt, J., \& Henning, V. (2009) Building successful online research networks with the last.fm model, Proceedings of the $5^{\text {th }}$ Open Knowledge Conference, 1-2.

Mendeley. (2009). Getting Started with Mendeley. Mendeley Desktop. London: Mendeley Ltd. Retrieved from http://www.mendeley.com. 


\section{Figures}

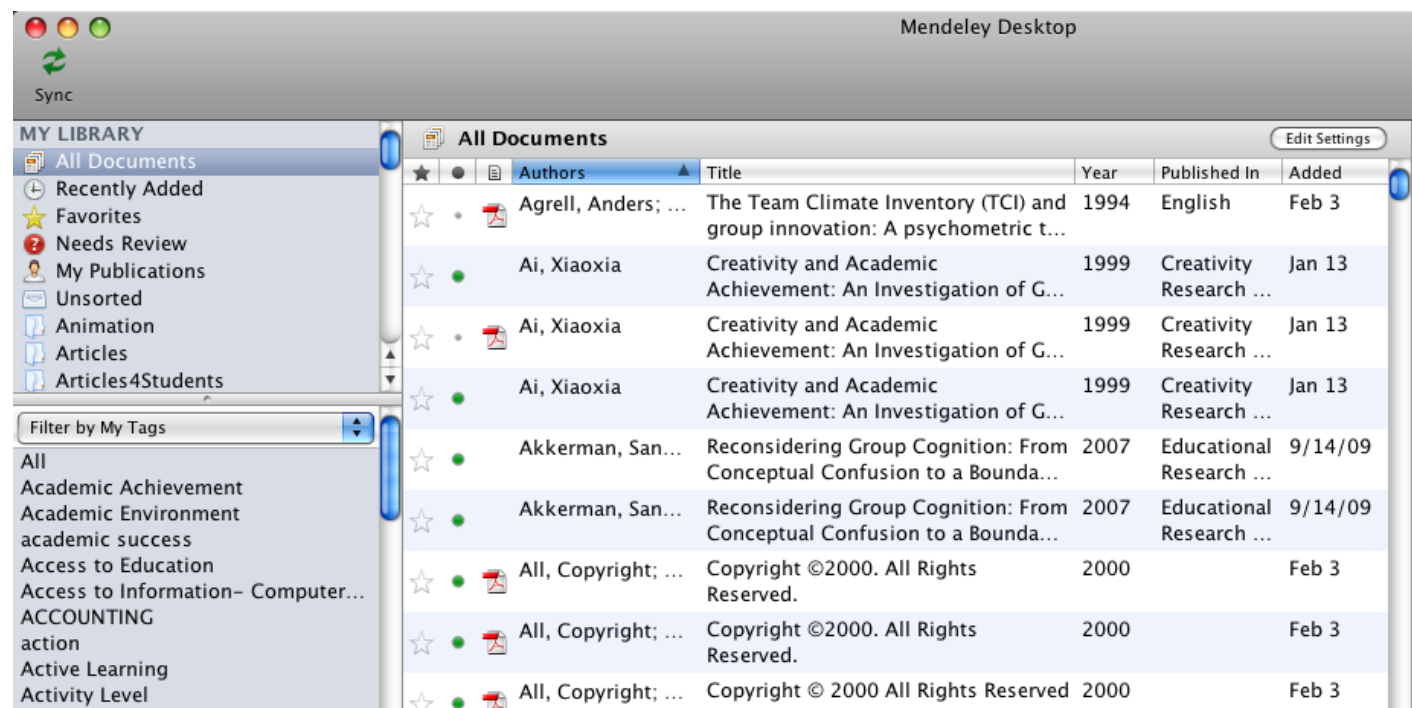

Figure 1. An image of Mendeley Desktop, showing the library, filter, and documents panes.

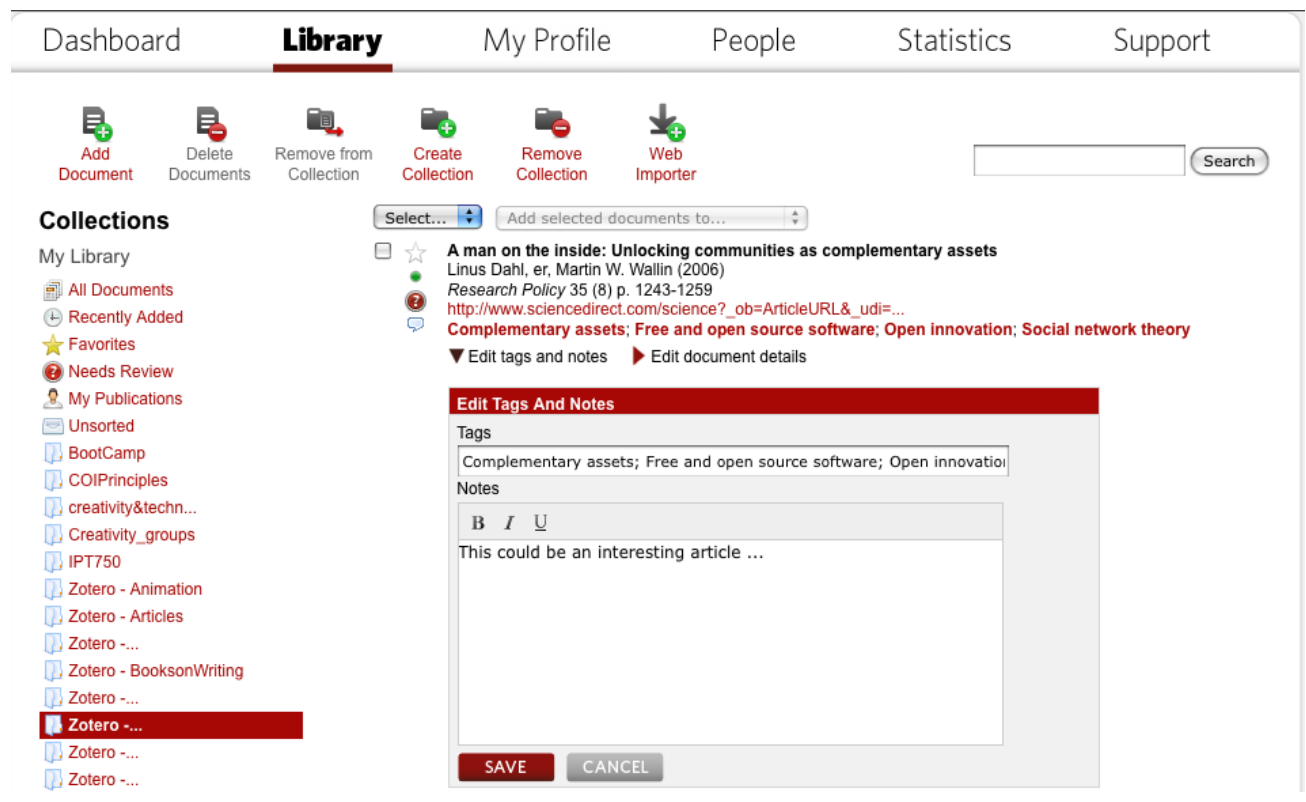

Figure 2. An image of Mendeley Online, showing the user's collections, citations, and notes/tags

fields. 


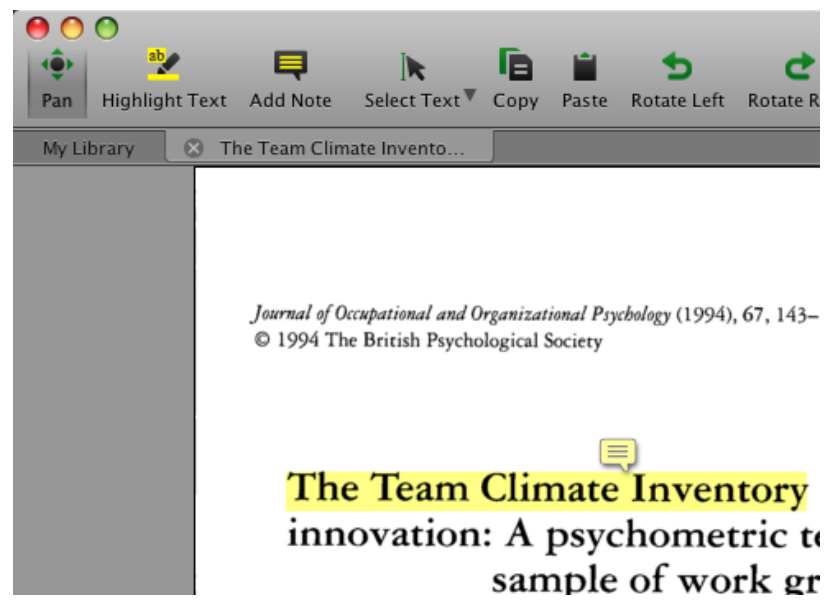

Figure 3. An image of Mendeley Desktop's PDF annotation and commenting tools.

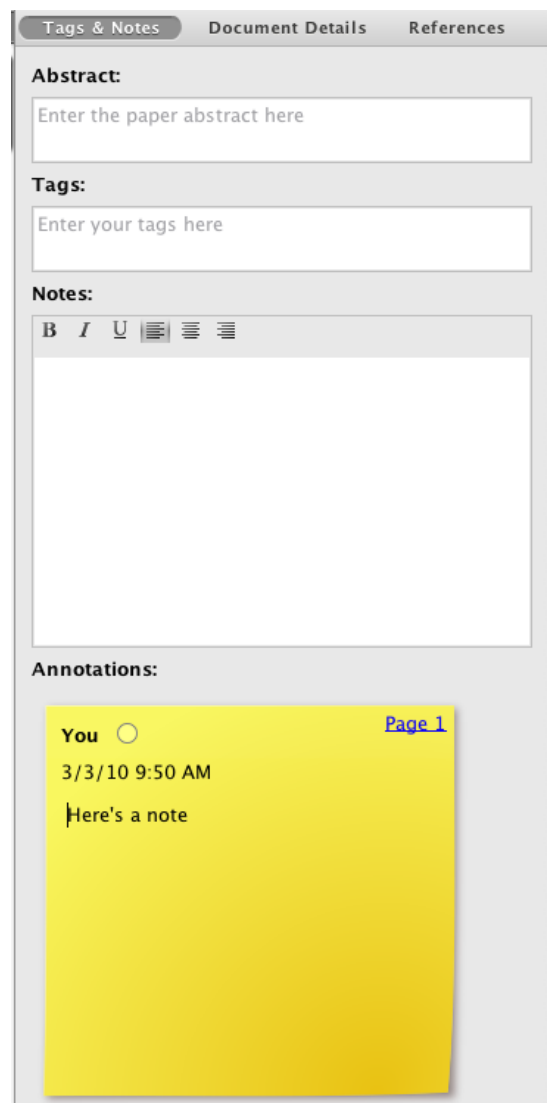

Figure 4. An image of Mendeley Desktop's Tags and Notes field, where a researcher can make whole-document notes or linked annotations. 